\title{
Interactive comment on "Dependence of the hygroscopicity parameter $\kappa$ on particle size, humidity and solute concentration: implications for laboratory experiments, field measurements and model studies" by Zhibin Wang et al.
}

\section{Anonymous Referee \#2}

\section{Received and published: 24 May 2017}

The study describes discrepancies of kappa values when derived over a range of water saturation (by HTDMA or CCNC measurements) as well as for different particles sizes and solute concentrations. The authors perform calculations for three substances, ammonium sulfate (AS), sodium chloride $(\mathrm{NaCl})$ and sucrose to demonstrate that varying results for kappa can be obtained based on the choice of input variables and then discuss their results together with findings from the literature. Main points of this work include that kappa needs to be carefully assessed especially for sub $10 \mathrm{~nm}$ particles because of kappa's size dependence; and that discrepancies in kappa can be due to calibrations that rely on different thermodynamic models. 
While this technical study elaborates on important points that should be taken into account, e.g. specifying the thermodynamic model used for instrument calibrations, it is also a study conducted in isolation lacking discussion on its relevance. Kappa is a useful parameter to represent particle hygroscopicity which is relevant for the number concentration of cloud condensation nuclei (CCN). The CCN number concentration is size dependent and smaller particles, especially sub $10 \mathrm{~nm}$, are significantly less relevant than larger particles. Based on this well-known fact the question comes up of how relevant the accuracy and precision of kappa for sub $10 \mathrm{~nm}$ particles is. This discussion is completely missing from the manuscript. Similarly, the title promises that this work discusses implications of varying kappa values for modeling studies. However, there is no discussion on the consequences and potential relevance of uncertainties in kappa values for any endpoint (CCN number concentration, cloud droplet number concentrations, cloud albedo, precipitation formation etc.) that might be considered in a model study.

Generally, among the points discussed the authors do not always distinguish clearly between the new contribution from their study and known aspects from previous publications. For example, a message of the paper is the concentration dependence of kappa (section 3.1) and the importance of the water activity. Already Petters and Kreidenweis (2007) have discussed this in their paper. It needs to be stated more clearly which is the new information that this work contributes and which aspects have been known beforehand. Against this background it is not fully clear which is the specific contribution the author would like to make with this work and why it is relevant.

In addition, at several occasions the authors seem to imply information without explicitly mentioning it, see specific comments section. This makes the manuscript difficult to understand and the messages are not always clear. Furthermore, the text needs to be revised for English grammar which also makes the text difficult to read. There are far too many mistakes to be pointed out at the review stage.

Printer-friendly version

I recommend that this manuscript undergoes major revisions based on the above and

Interactive

comment

\section{Discussion paper}

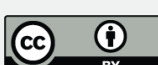


the more specific comments following below. It might also be worthwhile to rethink whether a "normal" scientific paper is the most appropriate type of manuscript, perhaps a "technical note" would be more appropriate.

Specific comments:

I. 21 - 24: How relevant are kappa closure studies for particles $<10 \mathrm{~nm}$ ? Why do particles $<10 \mathrm{~nm}$ deserve special attention in your manuscript?

I. $99 \mathrm{f}$ : The authors imply that $\mathrm{k} \_\mathrm{gf}$ is smaller than $\mathrm{k} \_c c n$ in normal cases which can be inferred from the statement "Conversely, higher $\bar{k} \_$gf compared to k_ccn...". However this is not stated explicitly. The text should clearly state what the more regular observation is and then explain differences from the norm. Also, it is not clear how often $k$ _f $>$ k_ccn has been found, because only one reference is provided. If there is only one study reporting this, the question is whether this is relevant information.

I. 105: Where does the information come from that kappa-composition relationships are only valid for particles $>50 \mathrm{~nm}$ ? A reference an explanation is missing.

I. 110: What exactly in multiphase chemistry needs to be understood with regards to kappa? It is not clear what the authors imply.

I. 111: How sensitive are climate modeling studies really to the accuracy of kappa? Particle hygroscopicity is only one factor among many that determine cloud properties. This argument needs to be more elaborated to make it convincing.

I. 154: This is confusing. In line $141 \mathrm{f}$ it is stated that literature values were used for sigma_sol, while here it is stated that the surface tension of water is used.

I. 175: An explanation for the Tolman effect is missing as well as a reference.

I. 188f: An explanation why kappa first decreases and then reaches a plateau is missing.

Printer-friendly version

I. 198ff: More elaboration of why kappa changes with size is needed given that gf 
remains constant. The authors should also highlight in how far this finding is new, no references are given in this paragraph, and how it relates to the observations in the field that kappa changes with size (e.g. Good et al. 2010, Jaatinen et al. 2014). The difference with the field observations is that the chemical composition changes with size and hence kappa. In the calculations performed in this work the composition and solution concentration remains the same, still kappa changes. Jaatinen, A., Romakkaniemi, S., Anttila, T., Hyvarinen, A. P., Hao, L. Q., Kortelainen, A., ... \& Laaksonen, A. (2014). The third Pallas Cloud Experiment: Consistency between the aerosol hygroscopic growth and CCN activity. Boreal environment research, 19, SS368-SS368.

I. 212: Provide references.

I. 224: An explanation is missing why there is no convergence found for sucrose at 200 $\mathrm{nm}$.

I. 231: Why is it especially important to consider a changing kappa for such small particles? As long as they are not relevant for cloud formation the accuracy of the kappa value is not particularly important.

I. 237ff: The authors state that k_ccn > k_gf has mostly been attributed to potential measurement uncertainty and non-ideality of the solution but cite only one study per aspect. This is no basis for generalizing. Either more studies need to be cited or the potentially various explanations need to be named, because the authors claim to provide an alternative explanation without showing that it really is an alternative. How can the authors know that the parameterization method in all the studies was the problem? Is there enough information in each study to come to that conclusion? If yes, a table that lists the parameterization used would be helpful to get an overview. If not, more information is needed how the authors trust in their judgement that the parameterizations for the calibrations are the crucial factor for discrepancies?

I. 270f: How do the authors decide that $k$ gf is the norm and k_ccn the deviation? And how do the authors know that it is correct to assume that all studies used the AP3 
model? As suggested above, a table of the reviewed literature on such background information would make the assumptions more transparent.

I. 273: In cases where the hygroscopic behavior is inherently different did the authors of the studies claim that closure was needed? In other words, between the lines the authors imply some misconception on hygroscopic behavior in some studies. More background information is needed for the reader to understand that.

Interactive

comment

I. 295f: What do the authors mean with the last two sentences on this page? Which are "such applications" and how is "self-consistent" defined?

I. 308: How many studies use an approach via kappa to estimate the fractions of organic and inorganic aerosol components? Is this an important application?

I. 311f: An explanation and a reference for the "nano-CCNC" are missing. Furthermore, it is not clear which particles with an unknown organic mass fraction the authors refer to. Ambient aerosol measured in the cited study, the $2.5 \mathrm{~nm}$ AS particles? Also, how do the authors derive the value of $50 \%$ uncertainty in organic mass fraction in case of inconsistent parameter use?

I. 329: Again, such small particles are hardly relevant as CCN. Reframe the statement.

Figure 1: Information is missing on where the solid lines are derived from.

Figure 4: It is difficult to distinguish the symbols and their sizes in the cloud of markers. Field and lab studies could be shown in separate panels to make individual studies more visible. Also, there is only very limited information provided on the nature of the aerosol studied in the field. More information should be provided to make the comparison meaningful.

Technical comments:

I. 39: replace "for" by "as"

I. 40: studies instead of study 
I. 61: delete "been"

I. 63: parameterizations

ACPD

I. 68: insert "a" before representative

I. 94: or the presence "of" slightly

and many more, please take care of the grammar before any resubmission.

Interactive comment on Atmos. Chem. Phys. Discuss., doi:10.5194/acp-2017-253, 2017. 